\title{
Coefficient body for nonlinear resolvents
}

\author{
Dedicated to Professor Yuri Kozitsky on the occasion of his 70th birthday
}

\begin{abstract}
This paper is devoted to the study of families of so-called nonlinear resolvents. Namely, we construct polynomial transformations which map the closed unit polydisks onto the coefficient bodies for the resolvent families. As immediate applications of our results we present a covering theorem and a sharp estimate for the Schwarzian derivative at zero on the class of resolvents.
\end{abstract}

\section{Introduction}

We denote the set of holomorphic functions on a domain $D \subset \mathbb{C}$ with values in another domain $D_{1} \subset \mathbb{C}$ by $\operatorname{Hol}\left(D, D_{1}\right)$. Also we denote $\operatorname{Hol}(D):=$ $\operatorname{Hol}(D, D)$, the set of all holomorphic self-mappings of $D$.

For any $f \in \operatorname{Hol}(D, \mathbb{C})$ and any $z \in D$, the initial value problem

$$
\left\{\begin{array}{l}
\frac{d u}{d t}+f(u(t))=0, \\
u(0)=z
\end{array}\right.
$$

has the unique solution for $t$ small enough. At the same time, if the derivative $f^{\prime}(z)$ does not take real negative values, then the functional equation

$$
w+r f(w)=z
$$

has the unique solution $w=J_{r}(z)$ for $r$ small enough.

2010 Mathematics Subject Classification. Primary 30C50; Secondary 30D05.

Key words and phrases. Holomorphic function, infinitesimal generator, nonlinear resolvent, Schur parameter. 
Surprisingly, the problems (1.1) and (1.2) are closely connected. Namely, it was proved in [11] (see also $[4,15]$ ) that

$\diamond$ if $D$ is a bounded convex domain, then for every $f \in \operatorname{Hol}(D, \mathbb{C})$, the following two assertions are equivalent:

- for every $z \in D$, the initial value problem (1.1) has the unique solution (denoted by $u(t, z)$ ) on the positive half-axis $t \geq 0$ and $u(t, \cdot) \in \operatorname{Hol}(D)$;

- for every $z \in D$, the functional equation (1.2) has the unique solution $w=J_{r}(z)$, which is well defined for all $r \geq 0$ and $J_{r} \in \operatorname{Hol}(D)$.

If either of these conditions holds, then the family $\{u(t, \cdot)\}_{t \geq 0}$ forms a one-parameter semigroup on $D$. In this case, the function $f$ is called the (infinitesimal) generator of the semigroup $\{u(t, \cdot)\}_{t \geq 0}$. The function $J_{r}$ is called the nonlinear resolvent of the generator $f$, and the family $\left\{J_{r}\right\}_{r \geq 0}$ is called the resolvent family of $f$.

In view of the above equivalence, nonlinear resolvents serve as an important tool in the study of semigroups of holomorphic mappings. Bearing in mind this goal, various properties of a nonlinear resolvent were established; among them resolvent identities, exponential formula, asymptotic behaviour, etc. (see, for example, [15]).

At the same time, in general, nonlinear resolvents themselves are of independent interest since they are solutions of an important class of functional equations. From this point of view, the following question is principal:

- How to determine which functions appear to be resolvents?

To the best of our knowledge, only some necessary conditions are known. To present one of them, notice that a point $z_{0} \in D$ is the fixed point of the resolvent family $\left\{J_{r}\right\}_{r \geq 0}$ (that is, $J_{r}\left(z_{0}\right)=z_{0}$ for all $r \geq 0$ ) if and only if $f\left(z_{0}\right)=0$; see $[15,12]$ for details. Concerning resolvent families in the open unit disk $\mathbb{D}=\{z:|z|<1\}$, the following properties were recently shown in $[5]$ :

$\diamond$ Let $\left\{J_{r}\right\}_{r \geq 0}$ be the resolvent family of a generator $f$ on $\mathbb{D}$ with $f(0)=0$, that is,

$$
J_{r}=(I+r f)^{-1} \in \operatorname{Hol}(\mathbb{D}), \quad r \geq 0 .
$$

Then for each $r \geq 0$, the resolvent $J_{r}$ is a hyperbolically convex function (see, for example, [10]) and belongs to the Noshiro-Warschawski class (that $i s$, $\operatorname{Re} J_{r}^{\prime}(z)>0$ for all $\left.z \in \mathbb{D}\right)$. However, there are hyperbolically convex functions of the Noshiro-Warschawski class that are not resolvents.

Being holomorphic functions in the open unit disk, resolvents can be completely determined by their Taylor coefficients. The aim of this paper is to describe the coefficient bodies for the set of nonlinear resolvents. For this purpose we use the classical Faà di Bruno formula and Schur parameters on the one hand, and very recent results obtained in [9], on the other hand. 


\section{Preliminaries}

2.1. Bell polynomials and Faà di Bruno formula. The following construction is familiar in combinatorics and has various applications (see, for example, $[2,13])$.

Definition 2.1. Let $k \leq n$. The exponential Bell polynomials are given by

$$
B_{n, k}\left(x_{1}, x_{2}, \ldots, x_{n-k+1}\right)=n ! \sum_{j \in I_{k}} \prod_{i=1}^{n-k+1} \frac{1}{j_{i} !}\left(\frac{x_{i}}{i !}\right)^{j_{i}},
$$

likewise, the ordinary Bell polynomials are given by

$$
B_{n, k}^{o}\left(x_{1}, x_{2}, \ldots, x_{n-k+1}\right)=k ! \sum_{j \in I_{k}} \prod_{i=1}^{n-k+1} \frac{x_{i}^{j_{i}}}{j_{i} !},
$$

where $I_{k}$ consists of all multi-indexes $j=\left(j_{1}, \ldots, j_{n-k+1}\right)$ such that

$$
j_{1}, \ldots, j_{n-k+1} \geq 0, \quad \sum_{i=1}^{n-k+1} i j_{i}=n, \quad \sum_{i=1}^{n-k+1} j_{i}=k .
$$

For instance, setting $k=1$, we see that the only non-negative integers which solve the system of equations $1 \cdot j_{1}+2 \cdot j_{2}+\ldots+n \cdot j_{n}=n$ and $j_{1}+\ldots+j_{n}=1$ are $j_{n}=1$ and $j_{1}=\ldots=j_{n-1}=0$. Thus, by Definition 2.1 we have

$$
B_{n, 1}^{o}(x)=B_{n, 1}(x)=\frac{n !}{1 !} \cdot \frac{x_{n}}{n !}=x_{n} .
$$

Similarly, setting $k=n$, we have $j_{1}=n$. Thus, $B_{n, n}^{o}(x)=B_{n, n}(x)=x_{1}^{n}$.

One of the applications of Bell's polynomials is a generalization of the chain rule to higher-order derivatives. Namely, in the holomorphic case the classical Faà di Bruno formula [6] (see also discussion in [7]) can be formulated in the form:

Theorem 2.1. Let function $g$ be holomorphic in a neighborhood of a point $z_{0}$, and function $h$ be holomorphic in a neighborhood of $w_{0}=g\left(z_{0}\right)$. Then

$$
\frac{d^{n}}{d z^{n}}(h \circ g)\left(z_{0}\right)=\sum_{k=1}^{n} h^{(k)}\left(w_{0}\right) B_{n, k}\left(g^{\prime}\left(z_{0}\right), g^{\prime \prime}\left(z_{0}\right), \ldots, g^{(n-k+1)}\left(z_{0}\right)\right) \text {. }
$$

It turns out that an analog of Faà di Bruno's formula holds for general derivations of holomorphic mappings, see [8, Lemma 4.2].

As an immediate consequence of Theorem 2.1, we get the following:

Corollary 2.1. Let functions $g \in \operatorname{Hol}(\mathbb{D})$ and $h \in \operatorname{Hol}(\mathbb{D}, \mathbb{C})$ have the Taylor expansions $g(z)=\sum_{n=1}^{\infty} a_{n} z^{n}$ and $h(z)=\sum_{n=1}^{\infty} b_{n} z^{n}$, respectively. 
Denote $h \circ g(z)=\sum_{n=1}^{\infty} c_{n} z^{n}$. Then

$$
c_{n}=\sum_{k=1}^{n} b_{k} B_{n, k}^{o}\left(a_{1}, a_{2}, \ldots, a_{n-k+1}\right) .
$$

Proof. Set $z_{0}=0$ in Theorem 2.1. Since $h^{(k)}(g(0))=h^{(k)}(0)=k ! b_{k}$, one calculates

$$
\begin{aligned}
B_{n, k}\left(g^{\prime}(0), g^{\prime \prime}(0), \ldots, g^{(n-k+1)}(0)\right) & =n ! \sum_{j \in I_{k}} \prod_{i=1}^{n-k+1} \frac{1}{j_{i} !}\left(\frac{g^{(i)}(0)}{i !}\right)^{j_{i}} \\
= & n ! \sum_{j \in I_{k}} \prod_{i=1}^{n-k+1} \frac{1}{j_{i} !}\left(a_{i}\right)^{j_{i}}=\frac{n !}{k !} B_{n, k}^{o}\left(a_{1}, a_{2}, \ldots, a_{n-k+1}\right) .
\end{aligned}
$$

Hence the assertion follows by Theorem 2.1.

2.2. Schur parameters and coefficient bodies. Since every function in $\operatorname{Hol}(\mathbb{D}, \mathbb{C})$ can be identified with its Taylor coefficients, the following notation is relevant. Let $\mathcal{F}$ be a subclass of $\operatorname{Hol}(\mathbb{D}, \mathbb{C})$, denote by $X_{n}(\mathcal{F})$ the coefficient body of order $n$ for $\mathcal{F}$, that is,

$$
X_{n}(\mathcal{F})=\left\{\left(a_{0}, \ldots, a_{n}\right): \exists f \in \mathcal{F}, f(z)=\sum_{k=0}^{n} a_{k} z^{k}+O\left(z^{n+1}\right)\right\} .
$$

Definition 2.2 ([14], see also [16]). Let $\psi \in \operatorname{Hol}(\mathbb{D})$ be not a constant. Denote by $\sigma$ the mapping acting on $\operatorname{Hol}(\mathbb{D})$ and defined by

$$
(\sigma \psi)(z)=\frac{1}{z} \cdot \frac{\psi(z)-\psi(0)}{1-\overline{\psi(0)} \psi(z)}
$$

Consider the sequence $\psi_{n}=\sigma^{n} \psi$. The numbers $\gamma_{n}=\psi_{n}(0), n=0,1,2, \ldots$, are called the Schur parameters, and the vector $\vec{\gamma}(\psi)=\left(\gamma_{0}, \gamma_{1}, \ldots\right)$ is called the Schur vector of $\psi$.

Consider the non-analytic polynomial transformation $\overrightarrow{F_{n}}(\mathbf{z})$ of $\mathbb{C}^{n}$ whose coordinates are defined inductively by

$$
\begin{aligned}
& F_{1}\left(z_{1}\right)=z_{1}, \\
& F_{m}\left(z_{1}, z_{2}, \ldots, z_{m}\right)=\left(1-\left|z_{1}\right|^{2}\right) F_{m-1}\left(z_{2}, \ldots, z_{m}\right) \\
& \quad-\overline{z_{1}} \sum_{k=2}^{m-1} F_{m-k}\left(z_{2}, \ldots, z_{m-k+1}\right) F_{k}\left(z_{1}, \ldots, z_{k}\right) \quad \text { for } m=2, \ldots, n .
\end{aligned}
$$

Assume that $\psi \in \operatorname{Hol}(\mathbb{D})$ has the Schur vector $\vec{\gamma}(\psi)=\left(\gamma_{0}, \gamma_{1}, \ldots\right)$ and the Taylor expansion

$$
\psi(z)=c_{0}+c_{1} z+c_{2} z^{2}+\ldots
$$


Lemma 2.2 in [9] states that $\left(c_{0}, \ldots, c_{n-1}\right)=\overrightarrow{F_{n}}\left(\gamma_{0}, \ldots, \gamma_{n-1}\right)$.

The following assertion is a slightly reformulated Proposition 4.2 in [9] and describes coefficient bodies for $\operatorname{Hol}(\mathbb{D})$.

Theorem 2.2. The coefficient body $X_{n-1}(\operatorname{Hol}(\mathbb{D})) \subset \mathbb{C}^{n}$ is a convex compact set. Moreover, $\vec{F}_{n}$ maps $\overline{\mathbb{D}}^{n}$ continuously onto $X_{n-1}(\operatorname{Hol}(\mathbb{D}))$ and satisfies $\overrightarrow{F_{n}}\left(\mathbb{D}^{n}\right)=\operatorname{Int} X_{n-1}(\operatorname{Hol}(\mathbb{D}))$ and $\overrightarrow{F_{n}}\left(\partial\left(\mathbb{D}^{n}\right)\right)=\partial\left[X_{n-1}(\operatorname{Hol}(\mathbb{D}))\right]$. In addition, $\vec{F}_{n}: \mathbb{D}^{n} \rightarrow \operatorname{Int} X_{n-1}(\operatorname{Hol}(\mathbb{D}))$ is a real analytic diffeomorphism but $\overrightarrow{F_{n}}$ is not injective on $\partial\left(\mathbb{D}^{n}\right)$.

To proceed, we consider the following subclasses of $\operatorname{Hol}(\mathbb{D}, \mathbb{C})$ :

$\diamond \mathcal{P}=\{p \in \operatorname{Hol}(\mathbb{D}, \Pi): p(0)=1, \operatorname{Re} p>0\}$ for the Carathéodory class.

$\diamond \mathcal{G}_{0}=\{f \in \operatorname{Hol}(\mathbb{D}, \mathbb{C}): f(z)=z p(z), p \in \mathcal{P}\}$.

It follows from the Berkson-Porta formula [1] (see $[4,15]$ for details) that $\mathcal{G}_{0}$ consists of all generators on $\mathbb{D}$ which satisfy $f(0)=0$ and $f^{\prime}(0)=1$.

Let $p \in \mathcal{P}$ have the Taylor series

$$
p(z)=1+2 \sum_{n=1}^{\infty} b_{n} z^{n} .
$$

In [9], the authors use the one-to-one correspondence

$$
p(z)=\frac{1+z \psi(z)}{1-z \psi(z)}, \quad z \in \mathbb{D},
$$

between the classes $\mathcal{P}$ and $\operatorname{Hol}(\mathbb{D})$ to construct a polynomial transformation

$$
\overrightarrow{T_{n}}\left(\gamma_{1}, \gamma_{2}, \ldots, \gamma_{n}\right)=\left(T_{1}\left(\gamma_{1}\right), T_{2}\left(\gamma_{1}, \gamma_{2}\right), \ldots T_{n}\left(\gamma_{1}, \gamma_{2}, \ldots, \gamma_{n}\right)\right),
$$

such that $b_{m}=T_{m}\left(\gamma_{1}, \gamma_{2}, \ldots, \gamma_{m}\right)$, where $\vec{\gamma}$ is the Schur vector of $\psi$. This construction provides the proof of the following result.

Theorem 2.3 (Theorem 4.1 in [9]). Let $n$ be a positive integer. The coefficient body $X_{n}(\mathcal{P})$ of order $n$ for the Carathéodory class $\mathcal{P}$ is expressed as $\{1\} \times 2 V_{n}$, where $V_{n}$ is a convex and compact body in $\mathbb{C}^{n}$. Moreover, there exists a continuous mapping $\overrightarrow{T_{n}}$ of $\overline{\mathbb{D}^{n}}$ onto $V_{n}$, which satisfies $\overrightarrow{T_{n}}\left(\overline{\mathbb{D}^{n}}\right)=\operatorname{Int} V_{n}$ and $\vec{T}_{n}\left(\partial \mathbb{D}^{n}\right)=\partial V_{n}$. In addition, $\vec{T}_{n}\left(\mathbb{D}^{n}\right)$ is a real analytic diffeomorphism but $\overrightarrow{T_{n}}$ is not injective on the boundary $\partial \overline{\mathbb{D}^{n}}$ of $\overline{\mathbb{D}^{n}}$ for $n=2,3, \ldots$

Thereby it enables us to describe the coefficient bodies for $\mathcal{G}_{0}$.

Corollary 2.2. For every $n \geq 2$, the coefficient body $X_{n}\left(\mathcal{G}_{0}\right)$ of order $n$ for the cone $\mathcal{G}_{0}$ is expressed as $\{(0,1)\} \times Y_{n-1}$, where $Y_{n-1}=2 V_{n-1}$ and $V_{n}$ is defined in Theorem 2.3. Hence, for $k=1,2, \ldots, n-1$, we have

(i) $Y_{k}=2 \vec{T}_{k}\left(\overline{\mathbb{D}^{k}}\right)$;

(ii) Int $Y_{k}=2 \vec{T}_{k}\left(\mathbb{D}^{k}\right)$ and $\partial Y_{k}=2 \vec{T}_{k}\left(\partial\left(\mathbb{D}^{k}\right)\right)$; 
(iii) $2 \vec{T}_{k}\left(\mathbb{D}^{k}\right)$ is a real analytic diffeomorphism;

(iv) $\vec{T}_{k}$ is not injective on $\partial \overline{\mathbb{D}^{k}}$ of $\overline{\mathbb{D}^{k}}$.

Proof. Indeed, let $f \in \mathcal{G}_{0}$. Then there exists $p \in \mathcal{P}$, such that $f(z)=z p(z)$. Write $f(z)=\sum_{k=0}^{\infty} f_{k} z^{k}$, then by (2.8) and (2.10) we have

$$
f_{0}=0, f_{1}=1 \text { and } f_{k+1}=2 T_{k}\left(\gamma_{1}, \gamma_{2}, \ldots \gamma_{k}\right) \text { for all } k \geq 1 \text {. }
$$

So, the set

$$
Y_{n-1}=\left\{\left(f_{2}, \ldots, f_{n}\right): \exists f \in \mathcal{G}_{0}, f(z)=z+\sum_{k=2}^{n} a_{k} z^{k}+O\left(z^{n+1}\right)\right\}
$$

coincides with $V_{n-1}$ from Theorem 2.3. Thus assertions (i)-(iv) hold.

\section{Main results}

3.1. Recursive formulae for Taylor coefficients. Let $f \in \mathcal{G}_{0}$ and $r>0$. The resolvent $w=J_{r}(z)=\sum_{k=0}^{\infty} a_{k} z^{k}$ is the solution of the functional equation (1.2), that is, $w+r f(w)=z$. This implies $a_{0}=0$. Differentiating, one can also see that $a_{1}=\frac{1}{1+r}$.

We now turn to searching for a general formula for $a_{k}$. In view of formula (2.9), it is natural to describe the Taylor coefficients of a nonlinear resolvent in terms of the Taylor coefficients of a self-mapping.

Theorem 3.1. Let $J_{r}$ be the nonlinear resolvent of the function $f \in \mathcal{G}_{0}$ having the form $f(z)=z \frac{1+z \psi(z)}{1-z \psi(z)}$ for some $\psi \in \operatorname{Hol}(\mathbb{D})$ with the Taylor expansion (2.7). Then the Taylor coefficients $a_{n}, n \geq 2$, of $J_{r}$ can be found as follows: $a_{2}=-\frac{2 r}{(1+r)^{3}} c_{0}$ and remaining by the recursive formula

$$
\begin{aligned}
a_{n+1}= & -\frac{2 r}{(1+r)^{2}} \sum_{k=1}^{n} c_{k-1} B_{n, k}^{o}\left(a_{1}, \ldots, a_{n-k+1}\right) \\
& -\sum_{k=2}^{n}\left(\frac{1-r^{2}}{2 r}\right)^{k-1} B_{n, k}^{o}\left(a_{2}, \ldots, a_{n-k+2}\right),
\end{aligned}
$$

where $B_{n, k}^{o}$ are the ordinary Bell polynomials defined by (2.1).

Proof. According to our assumptions, we can rewrite the resolvent equation (1.2) in the form

$$
w(1-w \psi(w))+r w(1+w \psi(w))=z(1-w \psi(w)),
$$

where $w=J_{r}(z)=\sum_{n=1}^{\infty} a_{n} z^{n}$. Equivalently,

$$
w \psi(w)=\frac{z-w(1+r)}{z-w(1-r)} .
$$


Define now $\tilde{w}:=\frac{w}{z}-\frac{1}{1+r}=\sum_{n=1}^{\infty} a_{n+1} z^{n}$. Substituting $w=z\left(\tilde{w}+\frac{1}{1+r}\right)$ in the last displayed formula, we get

$$
w \psi(w)=-\frac{(1+r)^{2}}{2 r} \cdot \frac{\tilde{w}}{1-\frac{1-r^{2}}{2 r} \tilde{w}} .
$$

Taking into account that both sides of (3.2) are compound functions of $w$ (or $\tilde{w}$ ), we can apply the Faà di Bruno formula in the form (2.4) twice and compare the respective coefficients. This together with (2.3) leads us to the equality

$$
\begin{aligned}
& \sum_{k=1}^{n} c_{k-1} B_{n, k}^{o}\left(a_{1}, \ldots, a_{n-k+1}\right) \\
& =-\frac{(1+r)^{2}}{2 r} \sum_{k=1}^{n}\left(\frac{1-r^{2}}{2 r}\right)^{k-1} B_{n, k}^{o}\left(a_{2}, \ldots, a_{n-k+2}\right) \\
& =-\frac{(1+r)^{2}}{2 r}\left[a_{n+1}+\sum_{k=2}^{n}\left(\frac{1-r^{2}}{2 r}\right)^{k-1} B_{n, k}^{o}\left(a_{2}, \ldots, a_{n-k+2}\right)\right] .
\end{aligned}
$$

We now express $a_{n+1}$ explicitly and complete the proof.

In the light of Theorem 2.3 and Corollary 2.2, it might be of independent interest to express the Taylor coefficients of a resolvent in terms of the coefficients of the corresponding generator.

Theorem 3.2. Let $J_{r}$ be the nonlinear resolvent of the function $f \in \mathcal{G}_{0}$ having the Taylor expansion $f(z)=z+\sum_{k=2}^{\infty} f_{k} z^{k}$. The Taylor coefficients $a_{n}, n \geq 2$, of $J_{r}$ can be calculated by the recursion:

$$
a_{n}=-\frac{r}{1+r} \sum_{k=2}^{n} f_{k} B_{n, k}^{o}\left(a_{1}, a_{2}, \ldots, a_{n-k+1}\right), \quad n \geq 2 .
$$

Proof. Denote $u(z)=z+r f(z)$. Then $u(0)=0, u^{\prime}(z)=1+r f^{\prime}(z)$ and $u^{(k)}(z)=r f^{(k)}(z)$ for all natural $k \geq 2$. Hence

$$
u^{\prime}(0)=1+r \text { and } u^{(k)}(0)=r f^{(k)}(0), \text { for } k \geq 2 .
$$

Since $w=J_{r}(z)$ is the solution of the functional equation

$$
u\left(J_{r}(z)\right)=z, \quad \text { we have } u^{\prime}\left(J_{r}(z)\right) J_{r}^{\prime}(z)=1 .
$$

To calculate the subsequent Taylor coefficients of $J_{r}$, we use Corollary 2.1 and the fact that $J_{r}(0)=0=u(0)$ in the following way

$$
\left.\frac{1}{n !} \cdot \frac{d^{n}}{d z^{n}} u\left(J_{r}(z)\right)\right|_{z=0}=\sum_{k=1}^{n} \frac{u^{(k)}(0)}{k !} B_{n, k}^{o}\left(a_{1}, a_{2}, \ldots, a_{n-k+1}\right) .
$$


Clearly, (3.5) implies that $\left.\frac{d^{n}}{d z^{n}} u\left(J_{r}(z)\right)\right|_{z=0}=0$ for $n \geq 2$, therefore

$$
u^{\prime}(0) B_{n, 1}^{o}\left(a_{1}, a_{2}, \ldots, a_{n}\right)=-\sum_{k=2}^{n} \frac{u^{(k)}(0)}{k !} B_{n, k}^{o}\left(a_{1}, a_{2}, \ldots, a_{n-k+1}\right) .
$$

Using now formulae (3.4) and (2.3), we get (3.3).

3.2. Polynomial mappings onto coefficient bodies. In this section we turn to the main question of the paper: how to describe the coefficient bodies for the class of all nonlinear resolvents associated to $\mathcal{G}_{0}$.

Given $r>0$, denote by $\mathcal{J}_{r}^{0}$ the class of resolvents associated with $\mathcal{G}_{0}$ (that is, the class of functions defined by (1.3) with $\left.f \in \mathcal{G}_{0}\right)$ and define

$$
\mathcal{J}^{0}:=\bigcup_{r>0} \mathcal{J}_{r}^{0}
$$

Let $n \in \mathbb{N}$. For any $J \in \mathcal{J}_{r}^{0}$ we have $J(0)=0$ and $J^{\prime}(0)=\frac{1}{1+r}$; this gives that $X_{1}\left(\mathcal{J}_{r}^{0}\right)$ is a singleton, namely, $X_{1}\left(\mathcal{J}_{r}^{0}\right)=\left\{\left(0, \frac{1}{1+r}\right)\right\}$ and there is a subset $U_{n}^{r} \subset \mathbb{C}^{n}$ such that $X_{n+1}\left(\mathcal{J}_{r}^{0}\right)=\left\{\left(0, \frac{1}{1+r}\right)\right\} \times U_{n}^{r}$. So the problem is to determine the set $U_{n}^{r}$.

Consider a polynomial transformation of $\mathbb{C}^{n}$

$$
\overrightarrow{Q_{n}^{r}}(\mathbf{z})\left(=\overrightarrow{Q_{n}^{r}}\left(z_{1}, \ldots, z_{n}\right)\right):=\left(Q_{1}\left(z_{1}\right), Q_{2}\left(z_{1}, z_{2}\right), \ldots, Q_{n}\left(z_{1}, \ldots, z_{n}\right)\right)
$$

with coordinates

$$
\begin{aligned}
& Q_{1}\left(z_{1}\right)=\frac{-2 r}{(1+r)^{3}} z_{1} \quad \text { and } \\
& Q_{m}\left(z_{1}, \ldots, z_{m}\right) \\
& =-\frac{2 r}{(1+r)^{2}} \sum_{k=1}^{m} z_{k} B_{m, k}^{o}\left(\frac{1}{1+r}, Q_{1}\left(z_{1}\right), \ldots, Q_{m-k}\left(z_{1}, \ldots, z_{m-k}\right)\right) \\
& \quad-\sum_{k=2}^{m}\left(\frac{1-r^{2}}{2 r}\right)^{k-1} B_{m, k}^{o}\left(Q_{1}\left(z_{1}\right), \ldots, Q_{m-k+1}\left(z_{1}, \ldots, z_{m-k+1}\right)\right)
\end{aligned}
$$

for $m=2, \ldots, n$.

Lemma 3.1. Let $\overrightarrow{Q_{n}^{r}}$ be defined by (3.7), then

(i) for any $\psi \in \operatorname{Hol}(\mathbb{D})$, the Taylor coefficients of the nonlinear resolvent of the function $f(z)=z \frac{1+z \psi(z)}{1-z \psi(z)}$ satisfy

$$
a_{m}=Q_{m-1}\left(c_{0}, c_{1}, \ldots, c_{m-2}\right),
$$

where $c_{m}$ are the Taylor coefficients of $\psi$ for all $m=2, \ldots, n+1$;

(ii) the mapping $\overrightarrow{Q_{n}^{r}}$ is an automorphism of $\mathbb{C}^{n}$ which maps $X_{n-1}(\operatorname{Hol}(\mathbb{D}))$ onto $U_{n}^{r}$. 
Proof. Assertion (i) follows from comparison of formula (3.1) in Theorem 3.1 with the construction of the transformation $\overrightarrow{Q_{n}^{r}}$ given in (3.7).

To prove (ii), define additional polynomial transformation by the following recursion:

$$
\begin{aligned}
& R_{1}\left(w_{1}\right)=\frac{-(1+r)^{3}}{2 r} w_{1} \quad \text { and } \\
& R_{m}\left(w_{1}, \ldots, w_{m}\right)=-\frac{-(1+r)^{m+2}}{2 r} w_{m} \\
& \quad-(1+r)^{m} \sum_{k=1}^{m-1} R_{k}\left(w_{1}, \ldots, w_{k}\right) B_{m, k}^{o}\left(\frac{1}{1+r}, w_{1}, \ldots, w_{m-k}\right) \\
& \quad-\frac{(1+r)^{m+1}}{1-r} \sum_{k=2}^{m}\left(\frac{1-r^{2}}{2 r}\right)^{k} B_{m, k}^{o}\left(w_{1}, \ldots, w_{m-k+1}\right) .
\end{aligned}
$$

for $m=2, \ldots, n$. A direct calculation shows that for every point $\mathbf{w} \in \mathbb{C}^{n}$ the equality $\mathbf{w}=\overrightarrow{Q_{n}^{r}}(\mathbf{z})$ is equivalent to $\mathbf{z}=\vec{R}_{n}(\mathbf{w})$. So, $\overrightarrow{Q_{n}^{r}}$ and $\overrightarrow{R_{n}}$ are polynomial automorphisms of $\mathbb{C}^{n}$.

Let $\psi \in \operatorname{Hol}(\mathbb{D})$ have the Taylor coefficients $\left(c_{0}, \ldots, c_{n-1}\right)=$ : c. By assertion (i), the vector $\overrightarrow{Q_{n}^{r}}(\mathbf{c})=: \mathbf{a}=\left(a_{2}, \ldots, a_{n+1}\right)$ consists of Taylor coefficients of the resolvent $J_{r}$; hence $\mathbf{a} \in U_{n}^{r}$. On the other hand, if $\mathbf{a} \in U_{n}^{r}$ then there is a function with the Taylor expansion

$$
\frac{1}{1+r} z+a_{2} z^{2}+\ldots+a_{n+1} z^{n+1}+o\left(z^{n+1}\right),
$$

which is a resolvent for some $f \in \mathcal{G}_{0}$ of the form $f(z)=z \frac{1+z \psi(z)}{1-z \psi(z)}$. Then the image $\overrightarrow{R_{n}}(\mathbf{a})$ gives the Taylor coefficients of $\psi$. The proof is complete.

Let the transformation $\overrightarrow{\Lambda_{n}^{r}}$ be defined by

$$
\overrightarrow{\Lambda_{n}^{r}}(\mathbf{z}):=\overrightarrow{Q_{n}^{r}} \circ \overrightarrow{F_{n}}\left(z_{1}, z_{2}, \ldots, z_{n}\right),
$$

where $\overrightarrow{F_{n}}$ is the real-analytic transformation defined in (2.6) (see also [9]).

Theorem 3.3. For any $r>0$ the coefficient body $X_{n+1}\left(\mathcal{J}_{r}^{0}\right)$ for $\mathcal{J}_{r}^{0}$ is $\left\{\left(0, \frac{1}{1+r}\right)\right\} \times U_{n}^{r}$, where $U_{n}^{r}=\vec{\Lambda}_{n}\left(\overline{\mathbb{D}^{n}}\right)$ is a compact set in $\mathbb{C}^{n}$.

Moreover, $\vec{\Lambda}_{n}$ is a continuous mapping of $\overline{\mathbb{D}^{n}}$ onto $U_{n}^{r}$, which satisfies $\vec{\Lambda}_{n}\left(\mathbb{D}^{n}\right)=\operatorname{Int} U_{n}^{r}$ and $\vec{\Lambda}_{n}\left(\partial\left(\mathbb{D}^{n}\right)\right)=\partial U_{n}^{r}$. In addition, $\vec{\Lambda}_{n}$ is a real analytic diffeomorphism on $\mathbb{D}^{n}$ but is not injective on the boundary of $\mathbb{D}^{n}$.

Proof. We already noted that $a_{0}=0$ and $a_{1}=\frac{1}{1+r}$. 
By Theorem 2.2, we have $\vec{F}_{n}\left(\overline{\mathbb{D}}^{n}\right)=X_{n-1}(\operatorname{Hol}(\mathbb{D}))$ and by assertion (ii) of Lemma 3.1, $\vec{Q}_{n}^{r}\left(X_{n-1}(\operatorname{Hol}(\mathbb{D}))\right)=U_{n}^{r}$. Therefore, the mapping $\overrightarrow{\Lambda_{n}^{r}}$ defined by (3.10) maps $\overline{\mathbb{D}}^{n}$ onto $U_{n}^{r}$.

Similarly to the proof of Theorem 4.1 in [9], we conclude that the other assertions follow from Theorem 2.2 because of the fact that the polynomial mapping $\overrightarrow{Q^{r}}$ defined by (3.7) is an automorphism of $\mathbb{C}^{n}$ by Lemma 3.1.

Now we turn to the coefficient body $X_{n+1}\left(\mathcal{J}^{0}\right)$. Observe that formula (3.6) implies that for any point $\mathbf{a} \in X_{n+1}\left(\mathcal{J}^{0}\right)$ we have $a_{0}=0$ and $a_{1} \in$ $(0,1)$. Hence, $X_{n+1}\left(\mathcal{J}^{0}\right)$ has no interior points and is not a closed set.

On the other hand, given $a \in(0,1)$, consider a polynomial transformation $\overrightarrow{\mathcal{Q}_{n}^{a}}$ of $\mathbb{C}^{n}$ defined by $\overrightarrow{\mathcal{Q}_{n}^{a}}=\overrightarrow{Q_{n}^{r}}$ with $r=\frac{1-a}{a}$. The mapping $\overrightarrow{\mathcal{Q}_{n}^{a}}$ is an automorphism of $\mathbb{C}^{n}$ and maps the set $X_{n-1}(\operatorname{Hol}(\mathbb{D}))$ onto the set $U_{n}^{\frac{1-a}{a}}$ by Lemma 3.1.

We now define one more transformation in $\mathbb{C}^{n}$ by the formula

$$
\overrightarrow{\Gamma_{n}^{a}}=\overrightarrow{\mathcal{Q}_{n}^{a}} \circ \overrightarrow{F_{n}} .
$$

This allows us to describe the coefficient body $X_{n}\left(\mathcal{J}^{0}\right)$ as follows.

Theorem 3.4. The coefficient body $X_{n+1}\left(\mathcal{J}^{0}\right)$ for $\mathcal{J}^{0}$ is $\{0\} \times \Upsilon_{n+1}$, where $\mathbf{a}=\left(a_{1}, \ldots, a_{n+1}\right) \in \Upsilon_{n+1}$ if and only if $a_{1} \in(0,1)$ and $\left(a_{2}, a_{3}, \ldots, a_{n+1}\right) \in$ $\vec{\Gamma}_{n}\left(\overline{\mathbb{D}^{n}}\right)$.

Moreover, for every $a \in(0,1)$ we have

(i) $\left\{\left(a_{2}, a_{3}, \ldots, a_{n+1}\right):\left(a, a_{2}, a_{3}, \ldots, a_{n+1}\right) \in \Upsilon_{n+1}\right\}=U_{n}^{\frac{1}{a}-1}$;

(ii) $\overrightarrow{\Gamma_{n}^{a}}$ is a continuous mapping of $\overline{\mathbb{D}^{n}}$ onto $U_{n}^{\frac{1}{a}-1}$;

(iii) $\overrightarrow{\Gamma_{n}^{a}}\left(\mathbb{D}^{n}\right)=\operatorname{Int} U_{n}^{\frac{1}{a}-1}$ and $\overrightarrow{\Gamma_{n}^{a}}\left(\partial\left(\mathbb{D}^{n}\right)\right)=\partial U_{n}^{\frac{1}{a}-1}$;

(iv) $\overrightarrow{\Gamma_{n}^{a}}$ is a real analytic diffeomorphism on $\mathbb{D}^{n}$ but is not injective on the boundary of $\mathbb{D}^{n}$.

This theorem follows immediately from Lemma 3.1 and Theorem 3.3 and the construction of the transformation $\overrightarrow{\Gamma_{n}^{a}}$.

The recursive formulas in the above theorems can work effectively and lead to interesting consequences.

Corollary 3.1. $X_{3}\left(\mathcal{J}^{0}\right)=\left\{\left(0, a_{1}, a_{2}\right): a_{1} \in(0,1), a_{2}=2 a_{1}^{2}\left(a_{1}-\right.\right.$ 1) $\gamma_{1}$ for some $\left.\gamma_{1} \in \overline{\mathbb{D}}\right\}$. Consequently, $\left|a_{2}\right| \leq 2 a_{1}^{2}\left(1-a_{1}\right)$.

As we mentioned in Section 1, every element of the resolvent family is a hyperbolically convex function. Without loss of generality, one assumes that $\arg a_{1}=0$ for a hyperbolically convex function $h, h(z)=a_{1} z+a_{2} z^{2}+\ldots$. It was shown by Ma and Minda in [10] that $\left|a_{2}\right| \leq a_{1}\left(1-a_{1}^{2}\right)$. Clearly, $2 a_{1}^{2}\left(1-a_{1}\right) \leq a_{1}\left(1-a_{1}^{2}\right)$, hence our estimate is better than the estimate for all hyperbolically convex functions (see Fig. 1). 


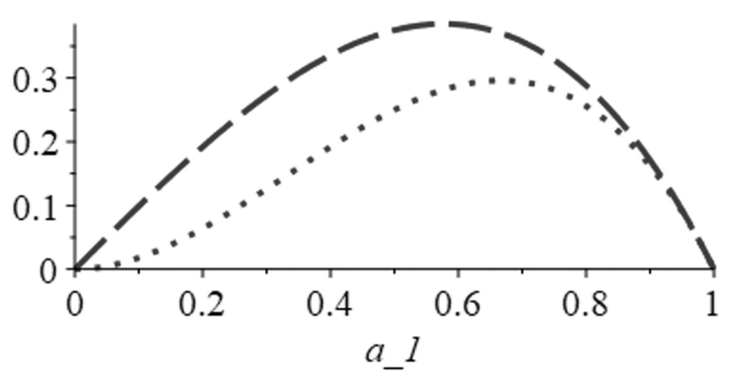

FiguRE 1. Estimates for $\left|a_{2}\right|$ for hyperbolically convex functions and for resolvents.

Corollary 3.2. $X_{4}\left(\mathcal{J}^{0}\right)=\left\{\left(0, a_{1}, a_{2}, a_{3}\right):\left(0, a_{1}, a_{2}\right) \in X_{3}\left(\mathcal{J}^{0}\right), a_{3}=\right.$ $2 a_{1}\left(a_{1}-1\right)\left[2 a_{2} \gamma_{1}+a_{1}^{2}\left(\gamma_{1}^{2}+\left(1-\left|\gamma_{1}\right|^{2}\right) \gamma_{2}\right)\right]$ for some $\left.\gamma_{1}, \gamma_{2} \in \overline{\mathbb{D}}\right\}$. Consequently, $a_{3}$ belongs to the disk with center at the point $\frac{\left(4 a_{1}-3\right) a_{2}^{2}}{2 a_{1}\left(a_{1}-1\right)}$ and of radius $\frac{4 a_{1}^{4}\left(1-a_{1}\right)^{2}-\left|a_{2}\right|^{2}}{2 a_{1}\left(1-a_{1}\right)}$.

These assertions can be obtained by direct calculations based on Lemma 3.1, Theorem 3.4 and the fact that the Schur parameters $\gamma_{1}$ and $\gamma_{2}$ lie in the closed unit disk.

The following covering result for resolvents is of independent interest.

Corollary 3.3. Let $J \in \mathcal{J}^{0}$. Then the image $J(\mathbb{D})$ contains the disk centered at the origin of radius $\frac{1}{2} \cdot \frac{a}{1+a(1-a)}$, where $a=J^{\prime}(0)$.

Proof. Assume that $w \notin J(\mathbb{D})$. Consider the function $g: \mathbb{D} \rightarrow \mathbb{C}$ defined $\operatorname{by} g(z)=\frac{w J(z)}{a(w-J(z))}$. Since $J$ is a univalent holomorphic function, then $g$ is. Then $g^{\prime}(0)=1$, so $\left|g^{\prime \prime}(0)\right| \leq 4$ (by the Biberbach inequality). Differentiating $g$ twice and using Corollary 3.1, we get

$$
\frac{2 a}{|w|}\left|1-2 w r \gamma_{1}(a-1)\right| \leq 4,
$$

which implies by the triangle inequality

$$
1-2|w|(1-a) \leq \frac{2|w|}{a} .
$$

So the result follows.

Remark that this result is not sharp (it can be seen letting $a \rightarrow 1^{-}$). In addition, Corollaries 3.1-3.2 imply a sharp estimation of the Schwarzian derivative at zero $\mathcal{S}_{J}(0)$ on the class of resolvents $\mathcal{J}^{0}$. 
Corollary 3.4. Let $J \in \mathcal{J}^{0}$. Then $\left|\mathcal{S}_{J}(0)\right| \leq \frac{16}{9}$. This estimate is sharp and is achieved for the resolvent $J_{1 / 2}$ corresponding to the infinitesimal generator $f(z)=z \frac{1+z^{2}}{1-z^{2}}$.

Proof. It follows by Corollaries 3.1-3.2 that there are $\gamma_{1}, \gamma_{2} \in \overline{\mathbb{D}}$ and $a \in$ $(0,1)$ such that

$$
\mathcal{S}_{J}(0)=12 a^{2}(a-1)\left[(2 a-1) \gamma_{1}^{2}+\left(1-\left|\gamma_{1}\right|^{2}\right) \gamma_{2}\right] .
$$

If $(2 a-1) \gamma_{1}=0$, the last factor takes maximal module 1 when $\gamma_{1}=0$ and $\gamma_{2}=1$. Otherwise, we can estimate it choosing $\arg \gamma_{2}=\arg \left((2 a-1) \gamma_{1}^{2}\right)$ and then $\left|(2 a-1) \gamma_{1}^{2}+\left(1-\left|\gamma_{1}\right|^{2}\right) \gamma_{2}\right| \leq|2 a-1|\left|\gamma_{1}\right|^{2}+1-\left|\gamma_{1}\right|^{2} \leq 1$. Therefore $\left|\mathcal{S}_{J}(0)\right| \leq 12 a^{2}(1-a)$. Maximizing the last expression with respect to $a \in(0,1)$, we obtain the desired result.

Notice that for functions of the Noshiro-Warschawski class (which contains $\mathcal{J}^{0}$; see [5]) the relevant estimate $\left|\mathcal{S}_{f}(0)\right| \leq 3$ is well known; for instance, it follows from a result in [3].

\section{Concluding remarks}

1. While the above results relate to resolvents associated to the class $\mathcal{G}_{0}$, they can be automatically extended to the class of generators normalized by $f(0)=0$ and $f^{\prime}(0)>0$. Indeed, denote the nonlinear resolvent corresponding to the infinitesimal generator $f$ by $J_{r}[f]$. Then $J_{r}[t f]=J_{t r}[f]$ for any $t>0$. In general, generators vanishing at zero satisfy $\operatorname{Re} f^{\prime}(0) \geq 0$. The study of this case needs a modified approach.

2. Another way to determine coefficient bodies for nonlinear resolvents is to use the Bürmann-Lagrange formula. At first glance, this seems even more natural. In fact, this leads to the same formulas as above, but requires more computations. An additional advantage of our method is that it can be slightly modified for the more general problem of finding coefficient bodies for the case where generators do not vanish at zero. We are supposed to do it somewhere else.

3. The above description of the coefficient bodies $X_{n+1}\left(\mathcal{J}_{r}^{0}\right)$ and $X_{n+1}\left(\mathcal{J}^{0}\right)$ was based on Theorems 2.2 (see also [9]) and 3.1. Alternatively, we can use Corollary 2.2 and Theorem 3.2. To this end, consider a polynomial transformation $\overrightarrow{L_{n}^{r}}(\mathbf{z})$ of $\mathbb{C}^{n}$ with coordinates $L_{1}\left(z_{1}\right)=\frac{-r}{(1+r)^{3}} z_{1}$ and

$$
\begin{aligned}
& L_{m}\left(z_{1}, \ldots, z_{m}\right) \\
& =\frac{-r}{1+r} \sum_{k=2}^{m+1} z_{k-1} B_{m+1, k}^{o}\left(\frac{1}{1+r}, L_{1}\left(z_{1}\right), \ldots, L_{m-k+1}\left(z_{1}, \ldots, z_{m-k+1}\right)\right)
\end{aligned}
$$

for $m=2, \ldots, n$. It can be shown that the transformation $\overrightarrow{L_{n}^{r}} \circ\left(2 \overrightarrow{T_{n}}\right)$ coincides with the transformation $\overrightarrow{\Lambda_{n}^{r}}$ above. 
4. It seems that similarly to Corollary 3.4, estimates of generalized Zalcman's and Fekete-Szegö's functionals can be treated.

Acknowledgment. The authors are very grateful to Prof. Toshiyuki Sugawa for useful discussions and comments.

\section{References}

[1] Berkson, E., Porta, H., Semigroups of analytic functions and composition operators, Michigan Math. J. 25 (1978), 101-115.

[2] Comtet, L., Advanced Combinatorics, D. Reidel Publishing Co., Dordrecht, 1974.

[3] Efraimidis, I., Vukotić, D., Applications of Livingston-type inequalities to the generalized Zalcman functional, Math. Nachr. 291 (2018), 1502-1513.

[4] Elin, M., Shoikhet, D., Linearization Models for Complex Dynamical Systems. Topics in univalent functions, functions equations and semigroup theory, Birkhäuser, Basel, 2010 .

[5] Elin, M., Shoikhet, D., Sugawa, T., Geometric properties of the nonlinear resolvent of holomorphic generators, J. Math. Anal. Appl. 483 (2020), Art. 123614.

[6] Faà di Bruno, F., Sullo sviluppo delle Funzioni, Annali di Scienze Matematiche e Fisiche 6 (1855), 479-480.

[7] Frabetti, A., Manchon, D., Five interpretations of Fa⿳亠 di Bruno's formula, IRMA Lect. in Math. and Theor. Phys. 21 (K. Ebrahimi-Fard and F. Fauvet, eds.), Europ. Math. Soc. (2015), 91-148.

[8] Kim, S.-A., Sugawa, T., Invariant differential operators associated with a conformal metric, Michigan Math. J. 55 (2007), 459-479.

[9] Li, M., Sugawa, T., Schur parameters and the Carathéodory class, Results in Mathematics, accepted for publication.

[10] Ma, W., Minda, D., Hyperbolically convex functions, Ann. Math. Polon. 60 (1994), $81-100$.

[11] Reich, S., Shoikhet, D., Metric domains, holomorphic mappings and nonlinear semigroups, Abstr. Appl. Anal. 3 (1998), 203-228.

[12] Reich, S., Shoikhet, D., Nonlinear Semigroups, Fixed Points, and the Geometry of Domains in Banach Spaces, World Scientific Publisher, Imperial College Press, London, 2005.

[13] Riordan, J., Combinatorial Identities, Robert E. Krieger Publishing Co., Huntington, NY, 1979.

[14] Schur, I., Methods in Operator Theory and Signal Processing, Operator Theory: Adv. and Appl. 18, Birkhäuser Verlag, 1986.

[15] Shoikhet, D., Semigroups in Geometrical Function Theory, Kluwer, Dordrecht, 2001.

[16] Simon, B., Orthogonal Polynomials on the Unit Circle, Part 1: Classical Theory, Colloquium Publications, Amer. Math. Society, 2005.

Mark Elin

Department of Mathematics

Ort Braude College

Karmiel 21982

Israel

e-mail: mark_elin@braude.ac.il
Fiana Jacobzon

Department of Mathematics

Ort Braude College

Karmiel 21982

Israel

e-mail: fiana@braude.ac.il

Received July 13, 2019 